\title{
DOA estimation of spectrally overlapped LFM signals based on STFT and Hough transform
}

\author{
Xiaofa Zhang, Weike Zhang ${ }^{*}$ (D) Ye Yuan, Kaibo Cui, Tao Xie and Naichang Yuan
}

\begin{abstract}
Traditional subspace methods which are based on the spatial time-frequency distribution (STFD) matrix have been investigated for direction-of-arrival (DOA) estimation of linear frequency modulation (LFM) signals. However, the DOA estimation performance may degrade substantially when multiple LFM signals are spectrally overlapped in time-frequency (TF) domain. In order to solve this problem, this paper proposes single-source TF points selection algorithm based on Hough transform and short-time Fourier transform (STFT). Firstly, the signal intersections in TF domain can be solved based on the Hough transform, and multiple-source TF points around the intersections are removed, so that the single-source TF points set is reserved. Then, based on the Euclidean distance operator, the single-source TF points set belonging to each signal can be obtained according to the property that TF points of the same signal have same eigenvector. Finally, the averaged STFD matrix is constructed for each signal, and DOA estimation is achieved based on multiple signal classification (MUSIC) algorithm. In this way, the proposed algorithm exhibit remarkable superiority in estimation accuracy and angular resolution over the state-of-the-art schemes and can achieve DOA estimation in the underdetermined cases. In addition, the proposed algorithm can still perform DOA estimation when multiple LFM signals intersect at one point. Numerical simulations demonstrate the validity of the proposed method.
\end{abstract}

Keywords: DOA estimation, LFM signals, TF analysis, STFT, Hough transform, Single-source TF points

\section{Introduction}

Direction-of-arrival (DOA) estimation is widely applied in array signal processing field, such as radar, wireless communications, sonar, and seismic sensing [1-4]. For the single-frequency signal, traditional subspace-based algorithms and their improved methods have been verified as efficient estimation techniques [5, 6]. With the rapid development of signal processing technologies, the linear frequency modulation (LFM) signal has many good characteristics, such as carrying a large amount of information and possessing better detection performance, which makes it being widely used in electronic countermeasures. Nevertheless, subspace-based algorithms have limitations to deal with LFM signals which are wideband and nonstationary; thus it is very important to study the

\footnotetext{
* Correspondence: xdwdz2010@163.com
State Key Laboratory of Complex Electromagnetic Environment Effects on

* Correspondence: xdwdz2010@163.com
State Key Laboratory of Complex Electromagnetic Environment Effects on Electronics and Information System, National University of Defense Technology, Changsha 410073, China
}

DOA estimation of LFM signals. The time-frequency (TF) analysis tools can deal with LFM signals, because they can make full use of the TF energy aggregation property of LFM signals. Belouchrani and Amin proposed the concept of STFD [7], and then a series of DOA estimation algorithms based on the STFD matrix have been proposed [8-14]. These algorithms mainly use the STFD matrix to replace the covariance matrix in the multiple signal classification (MUSIC) algorithm, which forms the TF-MUSIC algorithm. Compared with subspace-based algorithms, the TF-MUSIC algorithm can provide better DOA estimation performance because the noise power is spread while localizing the source energy in TF domain. In order to construct the STFD matrix, it is necessary to select the single-source TF points belonging to each signal in TF domain.

When LFM signals are TF-disjoint in TF domain, there exists efficient single-source TF points selection algorithms [15, 16]. The reference [15] obtained the 
single-source TF points based on the improved clustering algorithm. In [16], Heidenreich firstly applied the morphological image processing method to detect instantaneous frequency (IF) segments. Then, multiple detected IF segments of each signal are combined together for DOA estimation based on a bootstrap resampling technique. Unfortunately, these two methods only can be used in the situation that the signals are TF-disjoint. When the signals are non-disjoint (i.e., spectrally overlapped) in TF domain, the studies of single-source TF points selection algorithms are not very full. In [17-19], the single-source TF points selection algorithms are proposed based on the Wigner-Ville distribution (WVD). However, considering that the WVD has higher computational complexity and generates cross-term TF points in TF domain, it is very difficult to apply the WVD in practice. The reference [20] proposes the short-time Fourier transform (STFT) distribution model for DOA estimation of LFM signals, which greatly reduces implementation cost. Based on the STFT distribution, many DOA estimation algorithms have also been proposed [21-25] when LFM signals are non-disjoint in TF domain. However, all of them have some limitations. The single-source TF points selection algorithms in [21] are only suitable for real-valued array manifold matrix, but the array manifold vector in the DOA estimation is complex-valued. In [22, 23], the signals are required to satisfy the W-disjoint distribution in TF domain. Zhang proposes single-source TF points selection algorithm based on the TF points intensity information [24]. Although it can successfully realize the DOA estimation when two LFM signals are non-disjoint, the algorithm will fail when multiple LFM signals intersect at one point. In [25], the author uses the forward-backward spatial smoothing (FBSS) algorithm to eliminate the effect of multiple-source TF points and then perform DOA estimation. Although this method can achieve DOA estimation, the DOA estimation accuracy is poor.

In order to ease the limitations of existing algorithms for spectrally overlapped LFM signals, this paper propose the single-source TF points selection algorithm based on Hough transform and STFT when multiple LFM signals are non-disjoint in TF domain. Theoretically, the TF distribution of LFM signal is the straight line in TF domain. However, since the sampling data is discrete and finite, the TF distribution of each LFM signal under STFT has somewhat wide, which is called the TF ridgeline. Therefore, based on the Hough transform in TF domain, two line segments can be detected for every LFM signal. By traversing the TF domain, all line segments of LFM signals can be detected, and the line segments intersections can be calculated according to the endpoint coordinates. The area which is surrounded by these intersections is the multiple-source TF points set. Therefore, by removing multiple-source TF points around the intersections, the single-source TF points set is reserved. Then, based on the Euclidean distance operator, single-source TF points set belonging to each signal can be obtained according to the property that TF points of the same signal have same eigenvector. Finally, the averaged STFD matrix for every signal is computed, and DOA estimation can be achieved based on the MUSIC algorithm. In this way, the proposed algorithm has lower computational complexity and can achieve DOA estimation in the underdetermined cases. In addition, when there exists multiple signals at the intersections (i.e., greater than two), the accurate DOA estimation can still be achieved. Due to automatically select single-source TF points set of each signal, the proposed algorithm exhibits remarkable superiority in estimation accuracy and angular resolution over the state-of-the-art schemes.

The remainder of this paper is organized as follows. Section 2 formulates the STFT model of LFM signal based on the ULA and illustrates how to select singlesource TF points in detail. Section 3 carries the numerical simulations, and the proposed algorithm is discussed referring to the simulation results. Section 4 concludes the paper.

\section{Methods}

\subsection{STFT model of LFM signal}

In this paper, a uniform linear array (ULA) with $M$ sensors is considered, and the inter-element spacing is $d$. Furthermore, $N$ LFM signals are assumed to impinge on the ULA from $\theta_{1}, \theta_{2}, \cdots, \theta_{N}$. The array output at time $t$ can be modeled as

$$
\mathbf{X}(t)=\mathbf{A}(t) \mathbf{S}(t)+\mathbf{N}(t),
$$

where $\mathbf{X}(t)=\left[x_{1}(t), x_{2}(t), \cdots, x_{M}(t)\right]^{T}$ is the array observation vector, $\mathbf{S}(t)=\left[s_{1}(t), s_{2}(t), \cdots, s_{N}(t)\right]^{T}$ is the LFM signal vector, and $\mathbf{N}(t)=\left[n_{1}(t), n_{2}(t), \cdots, n_{M}(t)\right]^{T}$ is the additive white Gaussian noise vector. $\mathbf{A}(t)=\left[\mathbf{a}_{1}(t), \mathbf{a}_{2}(t), \cdots, \mathbf{a}_{N}(t)\right]$ is the $M \times N$ array manifold matrix, and $\mathbf{a}_{n}(t)=[$ $\left.\exp \left(-j f_{n}(t) \tau_{n 1}\right), \cdots, \exp \left(-j f_{n}(t) \tau_{n M}\right)\right]^{T}$ is the steering vector of the $n$th signal at time $t$, where $j$ is the imaginary unit. $\tau_{n m}=-2 \pi(m-1) d \cos \left(\theta_{n}\right) / c$ represents the time delay of the $n t h$ signal on the $m t h$ element respecting to the reference element, where $c$ is the speed of light. The instantaneous frequency $f_{n}(t)$ is denoted as

$$
f_{n}(t)=f_{n 0}+\gamma_{n} t
$$

where $f_{n 0}$ and $\gamma_{n}$ are initial frequency and modulated frequency of the $n t h$ signal, respectively. When $\gamma_{n}=0$, the LFM signal becomes narrowband single-frequency signal.

In 1946, Gabor proposed the concept of the STFT, which is an effective TF analysis tool for dealing with 
the LFM signal. The STFT distribution of $\mathbf{X}(t)$ in the free of noise can be expressed as

$$
\mathbf{S}_{X}(t, f)=\mathbf{A}(t) \mathbf{S}_{S}(t, f)=\mathbf{A}(t)\left[\begin{array}{c}
S_{S_{1}}(t, f) \\
\vdots \\
S_{S_{N}}(t, f)
\end{array}\right]
$$

where $S_{S_{n}}(t, f)$ is the STFT value of the $n t h$ LFM signal.

Then, the STFD matrix can be calculated through the correlation operation of $\mathbf{S}_{X}$, namely,

$$
\mathbf{D}_{X X}(t, f)=\mathbf{S}_{X} \mathbf{S}_{X}^{H}=\mathbf{A}(t) \mathbf{D}_{S S}(t, f) \mathbf{A}^{H}(t),
$$

where $\mathbf{D}_{X X}$ and $\mathbf{D}_{S S}$ are array output STFD matrix and signal STFD matrix, respectively.

The STFT distribution of LFM signals in TF domain can be described as Fig. 1. Figure 1a denotes that the LFM signals are TF-disjoint in TF domain, which means that signals are independent of each other, and the reference [15] has proposed efficient single-source TF points selection method.

This paper mainly studies that LFM signals are tiny spectrally overlapped in TF domain. That is to say, multiple signals only have a small number of same TF points in TF domain, as is shown in Fig. 1b. In the TF domain, there exist noise-term TF points and auto-term TF points, and auto-term contains single-source TF points and multiple-source TF points. Based on the proposed algorithm, we can remove noise-term TF points and multiple-source TF points in turn and obtain the singlesource TF points set of each signal. Finally, the STFD matrix can be constructed, and subsequent DOA estimation can also be achieved.

In TF domain, the LFM signals have obvious energy aggregation effect. According to [15], $\mathbf{D}_{X X}$ is very large at the TF ridgeline, and $\mathbf{D}_{X X}$ is close to zero at other TF points. Let $\Omega_{X}$ be the TF support domain, we can obtain

$$
\begin{array}{lc}
\mathbf{D}_{X X}(t, f) \gg 0 & (t, f) \in \Omega_{X} \\
\mathbf{D}_{X X}(t, f) \approx 0 & (t, f) \notin \Omega_{X} .
\end{array}
$$

In order to filter out the noise and obtain the TF points which have enough energy in TF domain, we can set an empirical threshold value and perform following criterion at each sampling time instant $(t, f)$ of $\mathbf{D}_{X X}$

$$
\frac{\left\|\mathbf{D}_{X X}(t, f)\right\|_{F}}{\max _{f}\left\|\mathbf{D}_{X X}(t, f)\right\|_{F}}>\varepsilon_{1} \quad(t, f) \in \Omega_{X},
$$

where $\|\cdot\|_{F}$ is the Frobenius norm and $\varepsilon_{1}$ is a small empirical threshold value and is related to noise. Typically, $\varepsilon_{1}=0.05$ when $\mathrm{SNR}=10 \mathrm{~dB}$ [18].

Then, the new TF points set $\Omega_{A}$ which includes single-source TF points $\Omega_{S}$ and multiple-source TF points $\Omega_{M}$ can be obtained.

\subsection{Extracting single-source TF points}

At the signal intersections, LFM signals are coherent owing to same frequency, which brings the rank deficiency of the signal subspace matrix. If these intersected TF points are selected to construct the STFD matrix, then subsequent DOA estimation will fail. Therefore, our goal is to remove multiple-source TF points and obtain single-source TF points set belonging to each signal.

In theory, two LFM signals only have an intersection in TF domain. However, since the sampling data is discrete and finite, the TF ridgeline of LFM signals under STFT has somewhat wide. Therefore, there exist multiple TF points around the intersections when LFM signals are non-disjoint in TF domain. By converting the TF distribution of LFM signals into ordinary grayscale image, we can use the Hough transform method to detect straight lines in the grayscale image [26]. Hough transform is a parameter estimation technique using the voting principle in TF domain. The basic principle is to map the straight line detecting problem in the plane coordinate domain into the parameter coordinate domain

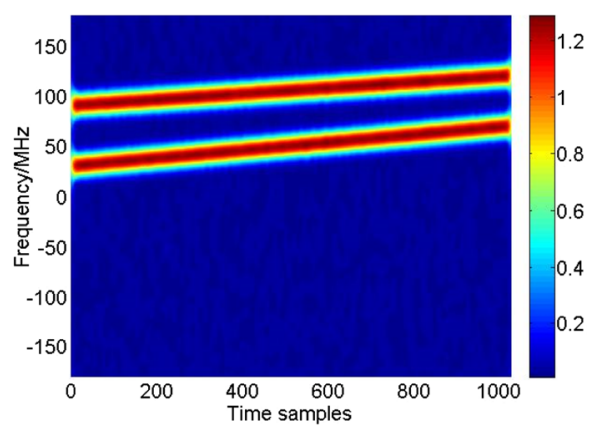

(a) TF-disjoint LFM signals

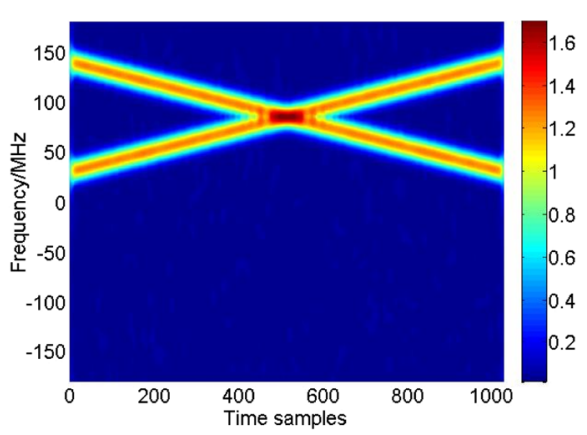

(b) TF non-disjoint LFM signals

Fig. 1 TF distribution condition of LFM signals, (a) TF-disjoint LFM signals, (b) TF non-disjoint LFM signals 
by using the point-line duality property, so that the mapped result is easier to be detected. It is assumed that the curve satisfies the following equation

$$
F\left((t, f),\left(a_{1}, a_{2}, \cdots, a_{m}\right)\right)=0 .
$$

In the plane Cartesian coordinates, the curve satisfies specific parameters $\left(a_{1}, a_{2}, \cdots, a_{m}\right)$, and $(t, f)$ is located at the curve. $\rho$ denotes the distance between the curve and the coordinate origin, and $\theta$ is the angle between $\rho$ and the sampling time axis. After mapping into the parameter domain, we use the polar coordinates to describe the parameters, and there exists following relationship

$$
\rho=t * \cos (\theta)+f * \sin (\theta) .
$$

Equation (8) shows that a point in the plane coordinates corresponds to a curve in the parameter coordinates. The different points of the same curve in the plane coordinates intersect at a point in the parameter coordinates, as is shown in Fig. 2.

Figure 2 shows that the three points $A, B, C$ of the same line in the plane coordinate domain can be mapped into three curves and curves intersect at the point $D$. By integrating along with different $\theta$, we can obtain the Hough transform. As a consequence, the Hough transform is a kind of projection integral essentially.

Firstly, we convert the TF distribution into the grayscale image. Then, by performing Hough transform on the grayscale image, we can obtain the corresponding curves of each TF points in the parameter domain. These curves exist multiple intersections, and each intersection corresponds to a detected line segment in the plane coordinate domain. For $N$ LFM signals in TF domain, $2 N$ peak points $\left(\theta_{1}, \rho_{1}\right),\left(\theta_{2}, \rho_{2}\right), \cdots,\left(\theta_{2 N}, \rho_{2 N}\right)$ can be searched by setting appropriate threshold value and carrying accumulation statistics; this is because the TF distribution of LFM signal under STFT has somewhat wide. The $2 N$ peak points correspond to $2 N$ line segments of the plane coordinate domain, and the

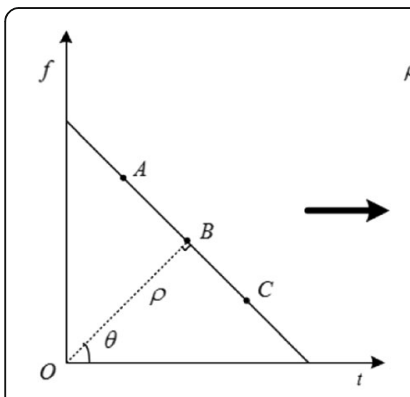

Plane coordinate domain

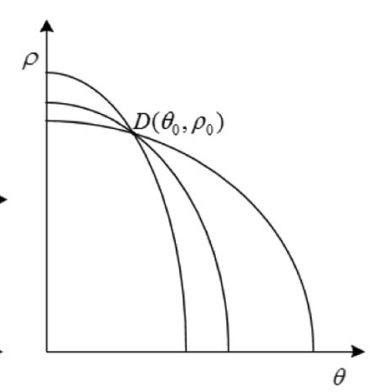

Parameter coordinate domain

Fig. 2 The Hough transform schematic mathematical equation of these lines can be calculated based on the endpoint coordinates, namely,

$$
\begin{gathered}
f_{1}=k_{1} t+b_{1} \\
\left\{\begin{array}{l}
f_{2}=k_{2} t+b_{2} \\
\vdots \\
f_{2 N}=k_{2 N} t+b_{2 N}
\end{array},\right.
\end{gathered}
$$

where $k_{1}, k_{2}, \cdots, k_{2 N}$ and $b_{1}, b_{2}, \cdots, b_{2 N}$ are slopes and intercepts of lines, respectively.

Since the noise in TF domain has been removed, the energy value in the TF ridgeline is much stronger than other TF points. Therefore, based on the Hough transform, we can precisely extract the line segments where TF distribution boundary of LFM signals is located in TF domain. Assuming that there exist $Q(Q \leq N(N-1) /$ 2) intersections between $N$ signals, then the detected $2 N$ line segments which corresponds to $N$ signals will have $P(P \geq 4 Q)$ intersections. If $N$ LFM signals only pairwise intersect, the detected line segments have $4 Q$ intersections. However, when there exists the situation that multiple signals intersect at one point, the number of intersections among $2 N$ line segments will be more than $4 Q$. For any intersection in TF domain, we can calculate all intersections $\left(t_{1}, f_{1}\right),\left(t_{2}, f_{2}\right), \cdots,\left(t_{P}, f_{P}\right)$ according to the line equation in the grayscale image. Then the minimum $\min \left(t_{1}, t_{2}, \cdots t_{P}\right)$ and maximum $\max \left(t_{1}, t_{2}, \cdots t_{P}\right)$ of the sampling time axis can be obtained in all intersections. In addition, the corresponding $\min \left(f_{1}, f_{2}, \cdots f_{U}\right)$ and $\max \left(f_{1}, f_{2}, \cdots f_{U}\right)$ can also be solved based on the (9), where $f_{1}, f_{2}, \cdots f_{U}$ are all frequency values that $\min \left(t_{1}, t_{2}\right.$, $\left.\cdots t_{P}\right)$ and $\max \left(t_{1}, t_{2}, \cdots t_{P}\right)$ correspond to detected line segments. If multiple-source TF points cannot be completely removed, these TF points are selected to estimate DOAs, which will result in the failure of DOA estimation. So we remove multiple-source TF points corresponding to the region of $\left(\min \left(t_{1} \cdots t_{P}\right), \max \left(t_{1} \cdots t_{P}\right)\right)$ and $\left(\min \left(f_{1} \cdots f_{U}\right), \max \left(f_{1} \cdots f_{U}\right)\right)$ and then obtain single-source TF points set $\Omega_{S}=\cup \Omega_{n}$ for $n=1,2, \cdots, N$, where $\Omega_{n}$ denotes single-source TF points set of the $n$th signal.

\subsection{Constructing STFD matrix}

In this subsection, we firstly calculate the single-source TF points set belonging to each signal and then construct the STFD matrix for subsequent DOA estimation. According to reference [15], for the signal STFD matrix $\mathbf{D}_{S S}(t, f)$, the diagonal elements have a larger value, and the remaining elements are close to zero at the singlesource TF points. As a result, based on the (4), the single-source TF points of the $n t h$ signal in $\Omega_{n}$ satisfies

$$
\mathbf{D}_{X X}(t, f)=\mathbf{D}_{S S}(t, f) \mathbf{a}_{n}(t) \mathbf{a}_{n}^{H}(t) \quad \forall(t, f) \in \Omega_{n} .
$$

For any two single-source TF points $\left(t_{i}, f_{i}\right)$ and $\left(t_{j}, f_{j}\right)$ in $\Omega_{n}$, (10) can be rewritten as 


$$
\begin{aligned}
& \mathbf{D}_{X X}\left(t_{i}, f_{i}\right)=\mathbf{D}_{S S}\left(t_{i}, f_{i}\right) \mathbf{a}_{n}(t) \mathbf{a}_{n}^{H}(t) . \\
& \mathbf{D}_{X X}\left(t_{j}, f_{j}\right)=\mathbf{D}_{S S}\left(t_{j}, f_{j}\right) \mathbf{a}_{n}(t) \mathbf{a}_{n}^{H}(t) .
\end{aligned}
$$

It can be seen from (10-12) that the STFD matrix $\mathbf{D}_{X X}$ of different TF points in $\Omega_{n}$ has same eigenvector $\mathbf{a}_{n}(t)$.

For any TF point $\left(t_{p}, f_{p}\right)$ in $\Omega_{S}$, we can compute the main eigenvalue $\lambda\left(t_{p}, f_{p}\right)$ and the corresponding principal eigenvector $\mathbf{e}\left(t_{p}, f_{p}\right)$ from the STFD matrix. Without loss of generality, we can make the first element of $\mathbf{e}\left(t_{p}, f_{p}\right)$ real and positive, namely,

$$
\tilde{\mathbf{e}}\left(t_{p}, f_{p}\right)=\left[\begin{array}{c}
\frac{\mathbf{e}_{1}\left(t_{p}, f_{p}\right)}{\left\|\mathbf{e}\left(t_{p}, f_{p}\right)\right\|} \\
\vdots \\
\frac{\mathbf{e}_{M}\left(t_{p}, f_{p}\right)}{\left\|\mathbf{e}\left(t_{p}, f_{p}\right)\right\|}
\end{array}\right] \cdot \frac{\left\|\mathbf{e}_{1}\left(t_{p}, f_{p}\right)\right\|}{\mathbf{e}_{1}\left(t_{p}, f_{p}\right)}
$$

where $\mathbf{e}_{m}\left(t_{p}, f_{p}\right)$ denotes the $m t h$ element of eigenvector $\mathbf{e}\left(t_{p}, f_{p}\right)$.

For single-source TF points set $\Omega_{S}$, mathematically, $\left(t_{i}, f_{i}\right)$ and $\left(t_{j}, f_{j}\right)$ belong to the same signal if they have following relationship

$$
d\left(\tilde{\mathbf{e}}\left(t_{i}, f_{i}\right), \tilde{\mathbf{e}}\left(t_{j}, f_{j}\right)\right)<\varepsilon_{2},
$$

where $d(\cdot)$ is the Euclidean distance operator between $\tilde{\mathbf{e}}($ $\left.t_{i}, f_{i}\right)$ and $\tilde{\mathbf{e}}\left(t_{j}, f_{j}\right) . \varepsilon_{2}$ is an empirical threshold value, which is greater than 0 . Typically, $\varepsilon_{2}=0.05$ when SNR = $10 \mathrm{~dB}$ [15]. By traversing entire set $\Omega_{S}$, we can obtain single-source TF points set $\Omega_{n}$ of the $n t h$ signal.

In order to reduce the calculation error and make full use of single-source TF points, we can compute the averaged STFD matrix of all TF points. For the nth signal, the averaged STFD matrix is expressed as

$$
\overline{\mathbf{D}}_{n}(t, f)=\frac{1}{\Sigma \Omega_{n}} \sum_{(t, f) \in \Omega_{n}} \mathbf{D}_{X X}(t, f),
$$

where $\overline{\mathbf{D}}_{n}(t, f)$ is similar to the covariance matrix in the subspace-based algorithm and $\Sigma \Omega_{n}$ denotes the number of single-source TF points.

Therefore, based on the MUSIC algorithm, we can carry eigendecomposition on the matrix $\overline{\mathbf{D}}_{n}(t, f)$ and obtain the signal subspace and noise subspace. Finally, we can construct the spectrum peak searching function and perform DOA estimation

$$
\mathbf{P}(\theta)=\frac{1}{\mathbf{b}^{H}(\theta) \mathbf{U}_{n} \mathbf{U}_{n}{ }^{H} \mathbf{b}(\theta)},
$$

where $\mathbf{b}(\theta)$ is the steering vector and $\mathbf{U}_{n}$ is the noise subspace.
For the readers' convenience, the procedure of singlesource TF points selection algorithm based on Hough transform and STFT is summarized as follows:

(1) Filter out noise according to empirical threshold value $\varepsilon_{1}$ in TF domain.

(2) Based on Hough transform, remove multiple-source TF points, and obtain all single-source TF points set $\Omega_{S}$.

(3) Classify signals according to the Euclidean distance operator, and get single-source TF points set $\Omega_{n}$ of each signal.

(4) For every signal, calculate the averaged STFD matrix $\overline{\mathbf{D}}_{n}(t, f)$ in the set $\Omega_{n}$, and then construct the spectrum peak search function $\mathbf{P}(\theta)=1 /$ $\mathbf{b}^{H}(\theta) \mathbf{U}_{n} \mathbf{U}_{n}{ }^{H} \mathbf{b}(\theta)$ based on the MUSIC algorithm.

\section{Results and discussion}

In order to verify the performance of the proposed algorithm, the following simulations are carried. Here, we set some general simulation parameters. The ULA has $M=$ 8 sensors, and the array element spacing is the half of the wavelength which corresponds to the lowest frequency of LFM signals. The sampling rate is $360 \mathrm{MHz}$ and snapshots are 1024. Since the STFT distribution can obviously gather the signal energy in TF domain and the SNR of signals is improved in TF domain by filtering the noise, we select SNR $=10 \mathrm{~dB}$ in the following analyses. Meanwhile, this paper involves the selection of two empirical threshold values. The threshold value $\varepsilon_{1}$ eliminates the effect of noise in TF domain, which is related to the SNR. We take $\varepsilon_{1}=0.05$ when SNR $=10 \mathrm{~dB}$, and $\varepsilon_{1}$ has been verified by [18]. Similarly, $\varepsilon_{2}=0.05$ when $\mathrm{SNR}=10 \mathrm{~dB}$, which is also verified by [15]. In addition, we proceed with the comparison simulations among STFT-MUSIC algorithm [20], STFT-FBSS-MUSIC algorithm [25], the reference [24], and the proposed algorithm. Firstly, the general performance of the proposed algorithm is verified, which includes the DOA estimation precision, the spatial resolution, and underdetermined DOA estimation, as is shown in Sections 3.1 and 3.2. Then, we verify the new property of the proposed algorithm, i.e., the DOA estimation performance when multiple LFM signals intersect at one point, as is shown in Section 3.3. In Section 3.4, the computational complexity of different algorithms is analyzed.

\subsection{DOA estimation accuracy analysis}

We consider that three spectrally overlapped LFM signals in TF domain come from- $30^{\circ}, 0,{ }^{\circ}$ and $30^{\circ}$. The frequency distribution of three signals are $[10,80] \mathrm{MHz}$, $[170,10] \mathrm{MHz}$, and $[60,170] \mathrm{MHz}$, respectively, and all signals have same power. Figure 3 illustrates the calculation process of the single-source TF points selection 


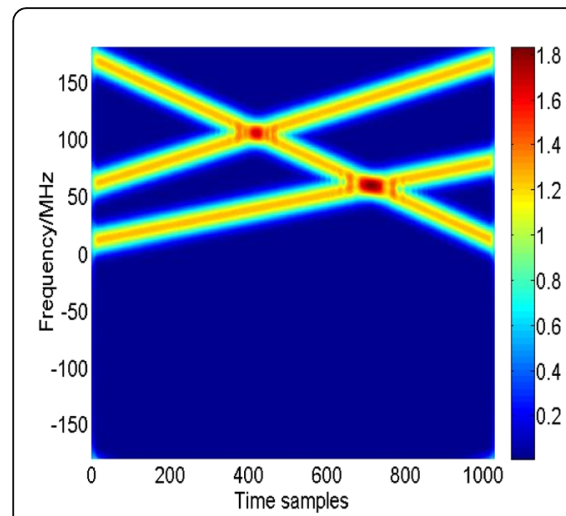

(a) The TF distribution

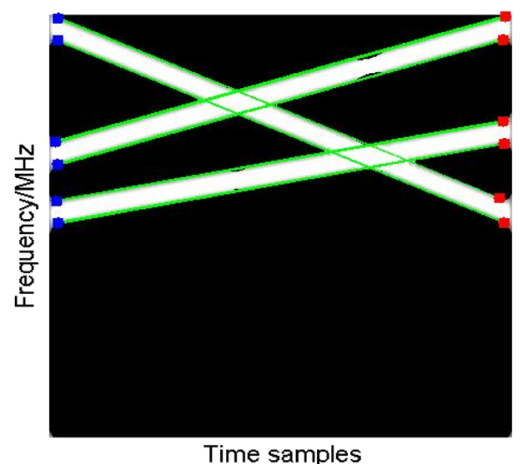

(d) The detected line segments

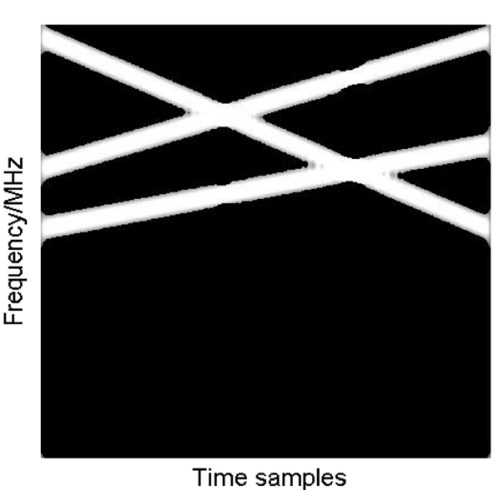

(b) The grayscale image

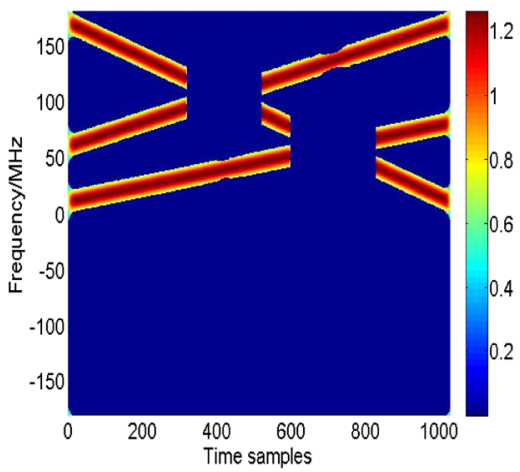

(c) Peak points in the parameter domain

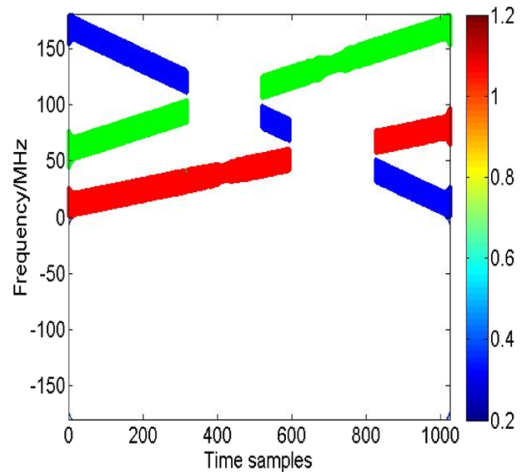

(e) Single-source TF points

Fig. 3 The single-source TF points selection method, (a) the TF distribution, (b) the grayscale image, (c) peak points in the parameter domain, (d) the detected line segments, (e) single-source TF points, (f) single-source TF points of each signal

method. Figure 3a displays the TF distribution of three LFM signals after filtering noise, whose grayscale image is showed in Fig. 3b. Based on Hough transform, we can search six peak points which is indicated by white in Fig. 3c, and Fig. 3d shows the detected six line segments. After removing multiple-source TF points, the single-source TF points set is shown in Fig. 3e. According to the Euclidean distance operator, we can classify signals and get single-source TF points set of each signal, as is shown in Fig. 3f.

Based on the abovementioned analysis, Fig. 4a plots ten typical independent estimated spatial spectra of the proposed algorithm, where the dashed lines represent the true DOAs. It can be seen that the proposed algorithm can accurately achieve DOA estimation. In order to quantitatively analyze the DOA estimation accuracy

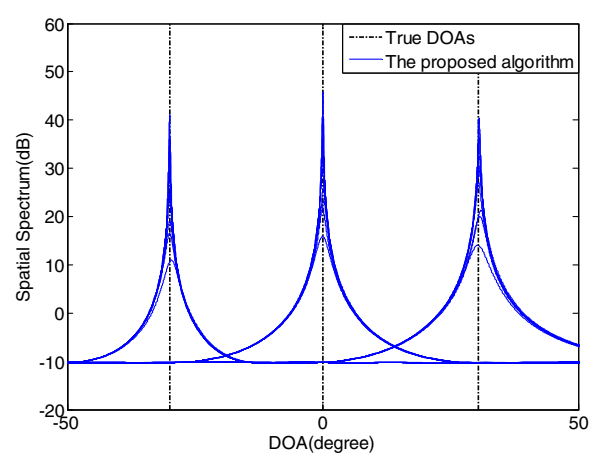

(a) The spatial spectra distribution

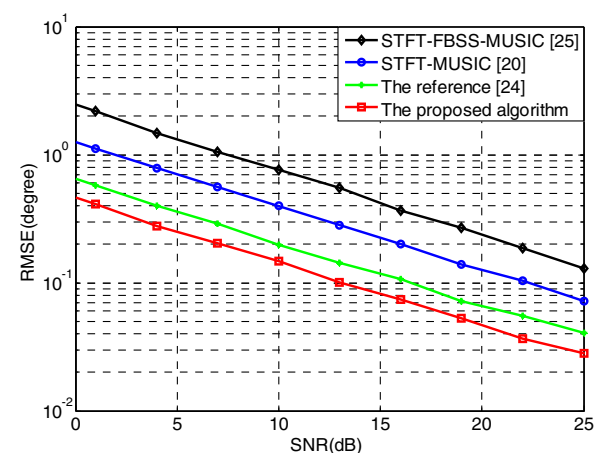

(b) The DOA estimation RMSE versus SNR

Fig. 4 The DOA estimation performance of three LFM signals, (a) the spatial spectrum distribution, (b) the RMSE of DOA estimation versus SNR 
of different algorithms, the root-mean-square error (RMSE) is introduced, which is denoted as

$$
\operatorname{RMSE}_{\theta}=\sqrt{\sum_{p=1}^{P} \sum_{n=1}^{N}\left(\hat{\theta}_{m n}-\theta_{n}\right)^{2} / M N}
$$

where $M$ is the number of Monte Carlo trials, $N$ is the number of signals, and $\hat{\theta}_{m n}$ is the estimated value of $\theta_{n}$ in the $m$ th simulation.

By carrying 1000 times independent Monte Carlo trials, Fig. 4b shows that the DOA estimation RMSE of 4 algorithms vary with the SNR. From Fig. 4b, we can see that DOA estimation RMSE of four algorithms decreases rapidly with the increase of SNR. In general, the STFTFBSS-MUSIC algorithm has the worst DOA estimation accuracy, and the estimation precision is $0.7594^{\circ}$ when SNR is $10 \mathrm{~dB}$. Because of performing the spatial smoothing operation, the array aperture of the STFT-FBSSMUSIC algorithm is reduced. As a result, the estimation precision is reduced. Then, it is followed by the STFTMUSIC algorithm, and the estimation precision is $0.3970^{\circ}$ when SNR is $10 \mathrm{~dB}$. The STFT-MUSIC algorithm performs DOA estimation by using the TF points at the TF ridgeline, and only few TF points are used to estimate DOAs, which results in low estimation precision. In addition, it is not realistic to assume that the TF points are available at the TF ridgeline. By removing multiple-source TF points, the reference [24] and the proposed algorithm can obtain single-source TF points set belonging to each signal. Then, by computing the averaged STFD matrix of all TF points for every signal, the DOA estimation precision of these two methods is improved. Compared with the [24], the proposed algorithm removes multiple-source TF points based on Hough transform and reserves as many single-source TF points as possible. Therefore, the proposed algorithm possesses the best estimation precision, which can achieve $0.1474^{\circ}$ when SNR is $10 \mathrm{~dB}$.

\subsection{The angular resolution analysis and underdetermined DOA estimation}

In order to verify the angular resolution of the proposed algorithm, we consider two spectrally overlapped LFM signals coming from $-0.5^{\circ}$ and $0.5^{\circ}$. The frequency distribution of two signals are $[170,10] \mathrm{MHz}$ and $[10,170]$ $\mathrm{MHz}$, respectively. Figure $5 \mathrm{a}$ plots ten typical independent estimated spatial spectra of the proposed algorithm, which shows that the proposed algorithm can achieve DOA estimation when the spatial spacing of signals is 1 . By performing 1000 times Monte Carlo trials, Fig. 5b displays that the DOA estimation RMSE of LFM signals at different SNR, which can be seen that the proposed algorithm has better DOA estimation accuracy when the DOA spacing is $1^{\circ}$. Although the spatial spacing is very close, they are still distinctly distributed in TF domain. Therefore, the proposed algorithm has better angular resolution.

It is known that conventional subspace-based algorithms will fail in the underdetermined cases. However, the TF analysis can solve this problem. Based on the abovementioned simulation condition, we assume that four spectrally overlapped LFM signals come from- 15 , $-5^{\circ}, 5^{\circ}$, and $15^{\circ}$. Figure 6 a plots ten typical independent estimated spatial spectra of this case. Then, we reduce the number of sensors to $M=3$, and the incident signals remain unchanged; ten independent estimated spatial spectra is plotted in Fig. 6b. It is seen that the proposed algorithm can correctly estimate DOAs in the underdetermined cases. The reason is that the proposed algorithm separately handles each signal in TF domain. Although the number of signals is more than that of sensors, the proposed algorithm can still achieve DOA estimation.

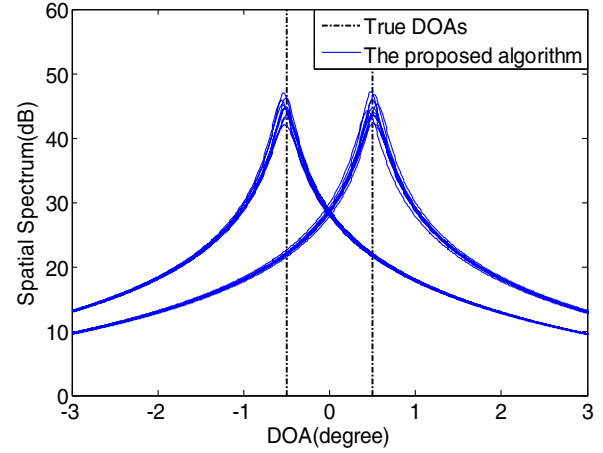

(a) The spatial spectra distribution

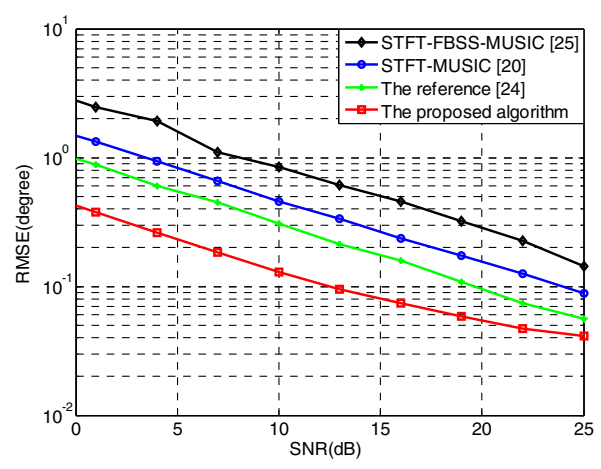

(b) The DOAestimation RMSE versus SNR

Fig. 5 The angular resolution of the proposed algorithm (DOA spacing: $1^{\circ}$ ), (a) the spatial spectrum distribution, (b) the RMSE of DOA estimation versus SNR 


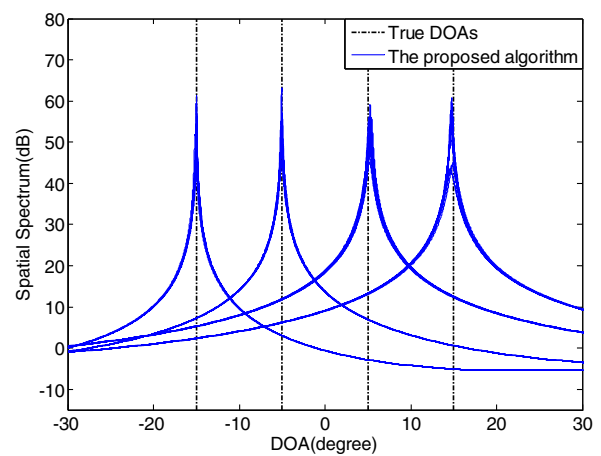

(a) $\mathrm{M}=8, \mathrm{~N}=4$

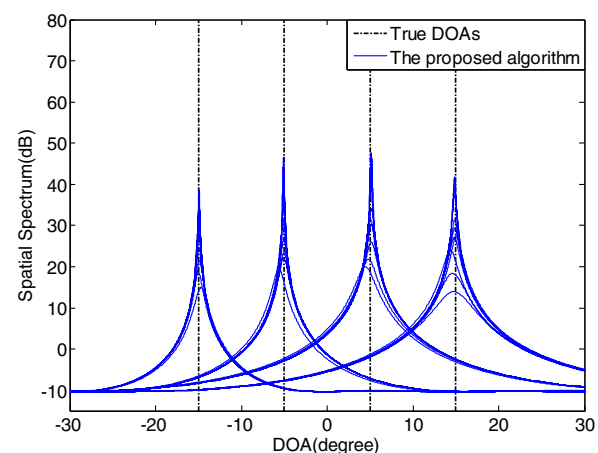

(b) $\mathrm{M}=3, \mathrm{~N}=4$

Fig. 6 The DOA estimation in the underdetermined cases, (a) $M=8, N=4$, (b) $M=3$, and $N=4$

According to Sections 3.1 and 3.2, we know that the STFT-MUSIC algorithm is not practical in the engineering applications and the DOA estimation precision of the STFT-FBSS-MUSIC algorithm is poor. Although the reference [24] has good DOA estimation accuracy, it is only applicable to the case that two LFM signals intersect at one point. When multiple signals intersect at one point, the algorithm will fail. However, the proposed algorithm is not restricted by this case. Based on the Hough transform, the proposed algorithm can achieve
DOA estimation when multiple signals intersect at one point. The following experiment will illustrate this property.

\subsection{The DOA estimation analysis when multiple LFM signals intersect at one point}

We consider that three spectrally overlapped LFM signals come from- $30^{\circ}, 0^{\circ}$, and $30^{\circ}$, and these signals intersect at one point in TF domain. The frequency distribution of three signals are $[170,10] \mathrm{MHz},[90,90] \mathrm{MHz}$

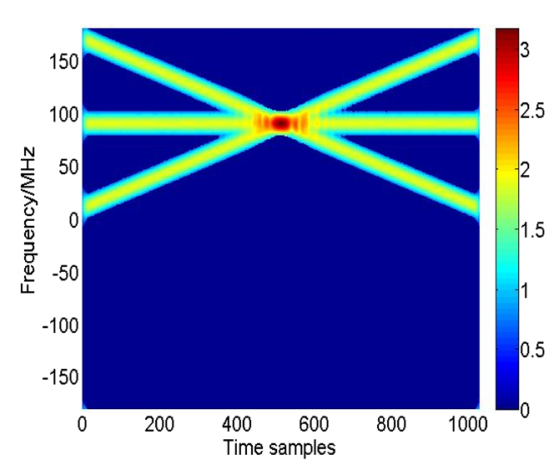

(a) The TF distribution of three signals

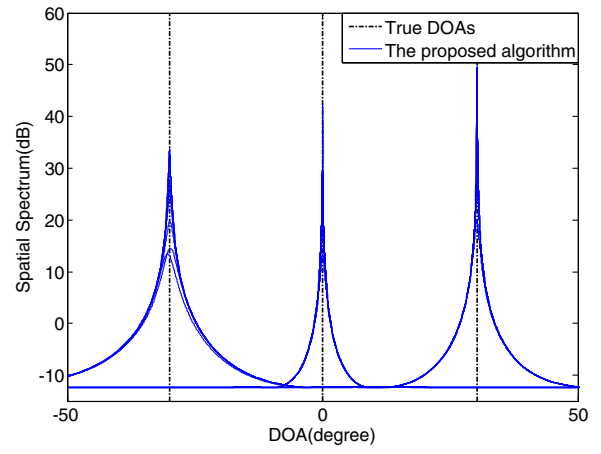

(c) The spatial spectra distribution

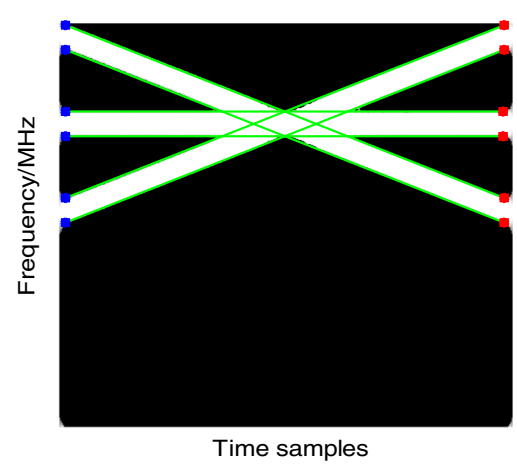

(b) The detected result based on Hough transform

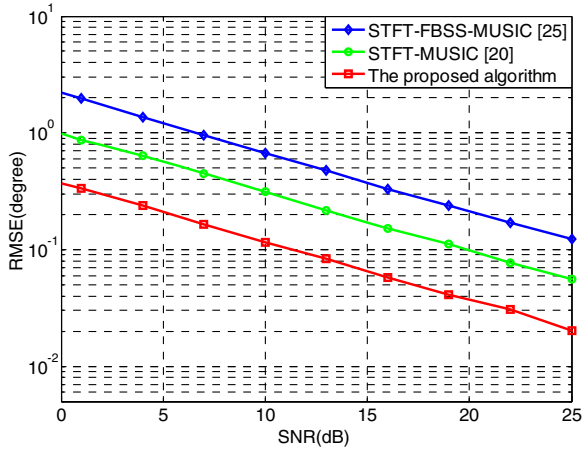

(d) The DOA estimation RMSE versus SNR

Fig. 7 The DOA estimation results analysis, (a) the TF distribution of three signals, (b) the detected result based on Hough transform, (c) the spatial spectra distribution, (d) the RMSE of DOA estimation versus SNR 
, and $[10,170] \mathrm{MHz}$, respectively. Other settings remain unchanged; the TF distribution of three signals is shown in Fig. 7a when SNR $=10 \mathrm{~dB}$, and Fig. 7b shows the detected results by using the Hough transform. Based on the proposed algorithm, the DOA estimation results are shown in Fig. 7c and d. It can be seen that the proposed algorithm has higher DOA estimation precision and the estimation precision can reach $0.1153^{\circ}$ when $\mathrm{SNR}=10$ $\mathrm{dB}$. Therefore, the proposed algorithm can achieve DOA estimation when multiple LFM signals intersect at one point.

\subsection{The computational complexity analysis}

The computational complexity comparison among the proposed algorithm and three existing methods are presented in Table. 1 . We consider a ULA with $M$ sensors and $N$ far-field LFM signals imping on the ULA. $L_{s}$ snapshots are used. In the STFT-FBSS-MUSIC algorithm, assuming that sub-array elements are $K(K<M)$. Considering that the TF-MUSIC algorithm is based on the quadratic TF operation, it has the highest computational complexity $O\left(N M^{2} L_{s}{ }^{2} \log _{2} L_{s}\right)$. Due to performing the FBSS algorithm, the STFT-FBSS-MUSIC algorithm possesses the lowest computational complexity $O\left(N K L_{s}{ }^{2} \log _{2} L_{s}\right)$. However, its estimation precision is poor. The reference [24] and the proposed algorithm have similar computational complexities, where $\Delta_{1}$ and $\Delta_{2}$ denote the increased calculation burden due to extract single-source TF points, respectively. $\Delta_{1}$ and $\Delta_{2}$ are similar and are much smaller than $O\left(N M L_{s}^{2} \log _{2} L_{s}\right)$. Therefore, the proposed algorithm has better estimation performance and acceptable computational complexity, which will have broad application prospects in the engineering.

\section{Conclusion}

Conventional DOA estimation algorithms will fail when LFM signals are spectrally overlapped in TF domain. In order to solve this problem, this paper proposes singlesource TF points selection algorithm based on Hough transform. For the purpose of reducing computational complexity, we select the STFT to analyze LFM signals. The proposed algorithm not only has higher DOA estimation precision and angular resolution but also can achieve DOA estimation in the underdetermined cases. In addition, the proposed algorithm can achieve DOA

Table 1 Comparison of computation complexity

\begin{tabular}{ll}
\hline Algorithm & Computation complexity \\
\hline TF-MUSIC [7] & $O\left(N M^{2} L_{s}{ }^{2} \log _{2} L_{5}\right)$ \\
STFT-FBSS-MUSIC [25] & $O\left(N K L_{s}{ }^{2} \log _{2} L_{s}\right)$ \\
The reference [24] & $O\left(N M L_{s}{ }^{2} \log _{2} L_{s}+\Delta_{1}\right)$ \\
The proposed algorithm & $O\left(N M L_{s}{ }^{2} \log _{2} L_{s}+\Delta_{2}\right)$ \\
\hline
\end{tabular}

estimation when multiple signals intersect at one point compared with existing algorithms. Although the current algorithm is only applicable to LFM signals which satisfy the straight line distribution in TF domain, generalized Hough transform can detect other forms of signals with different shapes in TF domain, such as sinusoidal FM signal and frequency-shift-keying signal. Therefore, the proposed algorithm has a broad application prospect in the electronic countermeasures.

\begin{abstract}
Abbreviations
DOA: Direction-of-arrivalLFMLinear frequency modulationSTFTShort-time Fourier transformSTFDSpatial time-frequency distributionTFTimefrequencyMUSICMultiple signal classificationFBSSForward-backward spatial smoothingULAUniform linear array
\end{abstract}

\section{Acknowledgements}

The authors would like to thank all anonymous reviewers and editors for their helpful suggestions for the improvement of this paper.

\section{Authors' contributions}

WZ and KC proposed the original idea of the full text; WZ and YY designed and implemented the simulation experiments; $W Z$ and TX wrote the manuscript under the guidance of NY; WZ and NY revised the manuscript; $X Z$ revised the manuscript and made great contribution to the final manuscript. All authors read and approved the final manuscript.

\section{Authors' information}

State Key Laboratory of Complex Electromagnetic Environment Effects on Electronics and Information System, National University of Defense Technology. No. 109, Deya Road, Kaifu District, Changsha, 410073, China.

\section{Funding}

This research received no specific grant from any funding agency in the public, commercial, or not-for-profit sectors.

\section{Availability of data and materials}

Data and materials are not possible to share publicly. If you have any interest in our data and materials, please contact the corresponding author by email.

Ethics approval and consent to participate

The manuscript does not study human and animals.

\section{Consent for publication}

The authors agree to publish the final manuscript.

\section{Competing interests}

The authors declare that they have no competing interests.

Received: 11 October 2018 Accepted: 20 November 2019

Published online: 05 December 2019

\section{References}

1. R. Schmidt, Multiple emitter location and signal parameter estimation. IEEE Trans. Antennas Propag. 34(3), 276-280 (1986)

2. R. Roy, T. Kailath, Direction-of-arrival estimation by subspace rotation methods-ESPRIT. IEEE Trans. Acoust. Speech. Signal Process. 37(7), 984-995 (1989)

3. SH Liu, YD Zhang, T Shan, Detection of weak astronomical signals with frequency-hopping interference suppression. Digit Signal Process. 72, 1-8 (2018)

4. WK Zhang, $X$ Chen, K Cui, T Xie, NC Yuan, DOA estimation for coprime linear array based on MI-ESPRIT and lookup table. Sennsors. 18(9), 3043 (2018)

5. SH Liu, T Shan, YD Zhang, R Tao, Y Feng, A fast algorithm for multicomponent LFM signal analysis exploiting segmented DPT and SDFrFT. In: Proceedings of the 2015 IEEE Radar Conference (RadarCon). 11391143 (2015) 
6. T. Shu, X. Liu, Robust and computationally efficient signal-dependent method for joint DOA and frequency estimation. EURASIP J. Adv. Signal Process. 2008(1), 1-16 (2008)

7. A. Belouchrani, M.G. Amin, Time-frequency MUSIC. IEEE Signal Process Lett. 6(5), 109-110 (2002)

8. A. Belouchrani, M.G. Amin, Blind source separation based on timefrequency signal representations. IEEE Trans. Signal Process. 46(11), 28882897 (1998)

9. M.G. Amin, Y.D. Zhang, Direction finding based on spatial time-frequency distribution matrices. Digit Signal Process. 10(4), 325-339 (2000)

10. Y.D. Zhang, W.-K. Ma, M.G. Amin, Subspace analysis of spatial timefrequency distribution matrices. IEEE Trans. Signal Process. 49(4), 747759 (2001)

11. A.B. Gershman, M. Pesavento, M.G. Amin, Estimating parameters of multiple wideband polynomial-phase sources in sensor arrays. IEEE Trans. Signal Process. 49(12), 2924-2934 (2001)

12. KB Cui, WW Wu, JJ Huang, X Chen, NC Yuan, DOA estimation of LFM signals based on STFT and multiple invariance ESPRIT. AEU-Int J Electron Commun. 77, 10-17 (2017)

13. J. Yu, L. Zhang, K. Liu, Coherently distributed wideband LFM source localization. IEEE Signal Process Lett. 22(4), 504-508 (2015)

14. A. Belouchrani, M.G. Amin, N. Thirion-Moreau, Y.D. Zhang, Source separation and localization using time-frequency distributions: an overview. IEEE Signal Process. Mag. 30(6), 97-107 (2013)

15. N. Linh-Trung, A. Belouchrani, K. Abed-Meraim, B. Boashash, Separating more sources than sensors using time-frequency distributions. EURASIP J. Appl. Signal Process. 2005(17), 2828-2847 (2005)

16. P. Heidenreich, L.A. Cirillo, A.M. Zoubir, Morphological image processing for FM source detection and localization. Signal Process. 89(6), 10701080 (2009)

17. E.M. Fadaili, N.T. Moreau, E. Moreau, Nonorthogonal joint diagonalization/ zero diagonalization for source separation based on time-frequency distributions. IEEE Trans. Signal Process. 55(5), 1673-1687 (2007)

18. A. Aissa-El-Bey, N. Linh-Trung, K. Abed-Meraim, A. Belouchrani, Y. Grenier, Underdetermined blind separation of nondisjoint sources in the timefrequency domain. IEEE Trans. Signal Process. 55(3), 897-907 (2007)

19. Y.D. Zhang, M.G. Amin, Blind separation of nonstationary sources based on spatial time-frequency distributions. EURASIP J. Adv. Signal Process. 2006(1), 1-13 (2006)

20. S.G. Kim, D.Y. Chang, Underdetermined blind source separation based on subspace representation. IEEE Trans. Signal Process. 57(7), 2604-2614 (2009)

21. Y. Li, S. Amari, A. Cichocki, D.W.C. Ho, S. Xie, Underdetermined blind source separation based on sparse representation. IEEE Trans. Signal Process. 54(2), 423-437 (2006)

22. A Jourjine, S Rickard, O Yilmaz, Blind separation of disjoint orthogonal signals: demixing sources from 2 mixtures. In: Proceedings of IEEE International Conference on Acoustics, Speech, and Signal Processing. 5, 29852988 (2002)

23. O. Yilmaz, S. Rickard, Blind separation of speech mixtures via time-frequency masking. IEEE Trans. Signal Process. 52(7), 1830-1847 (2004)

24. H.J. Zhang, G. Bi, Y. Cai, S.G. Razul, M.S.S. Chong, DOA estimation of closelyspaced and spectrally-overlapped sources using a STFT-based MUSIC algorithm. Digital Signal Process. 52, 25-34 (2016)

25. K.B. Cui, X. Chen, J.J. Huang, N.C. Yuan, DOA estimation of multiple LFM sources using a STFT-based and FBSS-based MUSIC algorithm. Radioengineering. 26(4), 1126-1137 (2017)

26. R. Duda, E. Peter, Use of the Hough transformation to detect lines and curves in pictures. Graphics and W. Newman Image Processing. 15(1), 11-15 (1972)

\section{Publisher's Note}

Springer Nature remains neutral with regard to jurisdictional claims in published maps and institutional affiliations.

\section{Submit your manuscript to a SpringerOpen ${ }^{\circ}$ journal and benefit from:}

- Convenient online submission

- Rigorous peer review

- Open access: articles freely available online

- High visibility within the field

- Retaining the copyright to your article

Submit your next manuscript at $\boldsymbol{\nabla}$ springeropen.com 